\title{
THE MEMBRANE OF INTACT HUMAN ERYTHROCYTES TOLERATES ONLY LIMITED CHANGES IN THE FATTY ACID COMPOSITION OF ITS PHOSPHATIDYLCHOLINE
}

\author{
F.A. KUYPERS, B. ROELOFSEN, J.A.F. OP DEN KAMP and L.L.M. VAN DEENEN \\ Laboratory of Biochemistry, State University of Utrecht, Padualaan 8, NL-3584 CH Utrecht (The Netherlands)
}

(Received July 22ńd, 1983)

Key words: Fatty acid composition; Phospholipid exchange protein; Osmotic fragility; (Erythrocyte membrane)

(1) Using the phosphatidylcholine specific transfer protein from bovine liver, native phosphatidylcholine from intact human erythrocytes was replaced by a variety of different phosphatidylcholine species without altering the original phospholipid and cholesterol content. (2) The replacement of native phosphatidylcholine by the disaturated species, 1,2-dipalmitoyl- and 1,2-distearoylphosphatidylcholine, proceeded at a low rate and extensive replacement could only be achieved by repeatedly adding fresh donor vesicles. The replacement by disaturated molecules was accompanied by a gradual increase in osmotic fragility of the cells, finally resulting in hemolysis when $40 \%$ of the native PC had been replaced. Up to this lytic concentration, the replacement did not affect the permeability of the membrane for potassium ions. (3) Essentially, all of the PC in the outer monolayer of the membrane could be replaced by 1-palmitoyl-2-oleoyl- and 1-palmitoyl-2-linoleoylphosphatidylcholine. These replacements did not alter the osmotic fragility of the cells, nor the $\mathrm{K}^{+}$ permeability of the membrane. (4) Increasing the total degree of unsaturation of the phosphatidylcholine species modified the properties of the membrane considerably. Replacement by 1,2-dilinoleoylphosphatidylcholine resulted in a progressive increase in osmotic fragility and hemolysis started to occur after $30 \%$ of the native PC had been replaced by this species. $\mathrm{K}^{+}$permeability was found to be slightly increased in this case. Cells became leaky for $\mathrm{K}^{+}$upon the introduction of 1-palmitoyl-2-arachidonoylphosphatidylcholine in the membrane. The increased permeability was also reflected by an apparent increase in the resistance of the cells against osmotic shock. (5) The conclusions to be drawn are that (i) 1-palmitoyl-2-oleoyl- and 1-palmitoyl-2-linoleoylphosphatidylcholine are species which fit most optimally into the erythrocyte membrane; (ii) loss of membrane stability results from an increase in the degree of saturation of phosphatidylcholine (unsaturation index $>0.5$ ) and (iii) the permeability is enhanced by increasing the content of highly unsaturated species (unsaturation index $>1.0$ ).

\section{Introduction}

The phospholipid composition of the erythrocyte membrane has been studied in great detail. The human erythrocyte membrane contains phosphatidylcholine (PC), phosphatidylethanolamine, phosphatidylserine and sphingomyelin as major

Abbreviation: PC, phosphatidylcholine. phospholipid constituents, minor amounts of phosphatidylinositol, phosphatidic acid and lysophospholipids, and furthermore a large quantity of cholesterol [1]. The fatty acid composition of each of the four major phospholipid classes has been clarified, while of PC, phosphatidylethanolamine and sphingomyelin even the fatty acyl species composition is known [2,3]. Finally, the distribution of the different phospholipid classes as well as of their molecular species over 
inner and outer layer of the membrane has been established [4-8,42]. Despite this detailed knowledge a number of problems remains unsolved so far. One of the questions is: why does the membrane contain so many different molecular species of a phospholipid like PC? And directly related to this: how far can one modify,i.e. by dietary means, the species composition of the PC before the structure and the function of the cell membrane becomes distorted? In this respect it is remarkable to notice how little variations are found in the overall phospholipid composition, and the fatty acid composition, under a variety of conditions. During the whole 120 days lifespan of the human erythrocyte, the overall phospholipid composition remains rather similar [1,9] and the differences in fatty acid composition which can be observed upon extreme variations in dietary fats are less pronounced than the changes in lipid composition of the plasma [10-13]. Relevant in this respect is the slow turnover of the membrane phospholipids: renewal of PC occurs by deacylation-reacylation as well as by an exchange of complete molecules with the serum; both processes proceed with a rate of about $1 \%$ per hour [14] and a halftime of $100 \mathrm{~h} \mathrm{[15],}$ respectively.

The composition of the erythrocyte appears to be highly conserved throughout, and it is obvious that this conservation serves the maintenance of specific membrane properties such as permeability and resistance against mechanical or osmotic stress. One way to investigate how and to what extent the various phospholipid classes contribute to the membrane properties, is to modify the structure or the content of one of the constituents and to record the resulting alterations. Since extensive modifications are impossible to obtain in vivo, an in vitro approach has been applied. Recently, Lange et al. [16] used the phosphatidylcholine specific transfer protein of bovine liver to modify the molecular species composition of $\mathrm{PC}$ in the rat erythrocyte membrane and it was observed that an increase in the degree of saturation of the phosphatidylcholine in this membrane resulted in hemolysis.

This approach has now been applied to study the human erythrocyte in which we followed alterations in osmotic fragility and $\mathrm{K}^{+}$permeability upon modifications in the fatty acyl content of the phosphatidylcholine. By using a variety of defined phosphatidylcholine species, it was possible to set the limits in between which the fatty acid composition of the phosphatidylcholine in the human erythrocyte membrane can be varied without drastic effects on membrane structure and function.

\section{Materials and Methods}

1,2-Dipalmitoyl-sn-glycero-3-phosphocholine and 1,2-dilinoleoyl-sn-glycero-3-phosphocholine, synthesized according to standardized procedures [17], were generously donated by members of the Biochemistry Department (Utrecht, The Netherlands). 1-Palmitoyl-2-oleoyl-sn-glycero-3-phosphocholine and egg phosphatidylcholine were purchased from Sigma (St. Louis, MO, U.S.A.); 1,2-distearoyl-sn-glycero-3-phosphocholine and 1,2-dioleoyl-sn-glycero-3-phosphocholine were purchased from Larodan (Malmö, Sweden). 1Palmitoyl-2-linoleoyl-L- $\alpha$-glycero-3-phosphocholine was obtained from P.L. Biochemicals (Milwaukee, U.S.A.); 1-palmitoyl-2-arachidonoyl-sn-glycero-3phosphocholine was obtained from Avanti Polar Lipids Inc. (Birmingham, AL, U.S.A.) and soybean phosphatidylcholine was kindly donated by Natterman (Köln, F.R.G.) Egg phosphatidyl$\left[N\right.$-methyl $\left.-{ }^{14} \mathrm{C}\right]$ choline and soybean phosphatidyl$\left[\mathrm{N}\right.$-methyl $\left.{ }^{14} \mathrm{C}\right]$ choline, synthesized according to Stoffel et al. [18], were generously donated by Dr. G. van Meer. Dipalmitoylphosphatidyl $N$-methyl-

${ }^{14} \mathrm{C}$ choline was purchased from New England Nuclear and Amersham International; 1-palmitoyl-2-[ $\left[{ }^{14} \mathrm{C}\right]$ oleoyl-sn-glycero-3-phosphocholine was obtained from Amersham. All other chemicals and solvents used were of PA-grade.

Preparation of vesicles. Vesicles were prepared from the various phosphatidylcholine species, mixed with an equimolar amount of cholesterol, 6 mol\% of egg phosphatidate (obtained by phospholipase D treatment of egg phosphatidylcholine), trace amounts of $\left[{ }^{14} \mathrm{C}\right]$ phosphatidylcholine and $\left[{ }^{3} \mathrm{H}\right]$ glycerol trioleate $(0.1 \mathrm{~mol} \%$ of total vesicle phosphatidylcholine). The lipid mixture was dried from a chloroform/methanol solution $(2: 1, \mathrm{v} / \mathrm{v})$. Vesicles were prepared by dispersing the dried lipid mixture above the transition temperature in a buffer containing $150 \mathrm{mM} \mathrm{NaCl}, 25 \mathrm{mM}$ glucose, $1 \mathrm{mM}$ EDTA $0.02 \%$ sodium azide $\left(\mathrm{NaN}_{3}\right)$ and 10 
$\mathrm{mM}$ Tris-HCl, $\mathrm{pH} 7.4$ (referred to as buffer throughout). The lipid dispersion was sonicated under nitrogen at 70 Watt in a Branson Sonifier for 3-6 minutes and centrifuged at $100000 \times g$ for $45 \mathrm{~min}$. Depending on the composition of the vesicles, the recovery varied from 50 to $85 \%$ and was determined by radioactivity measurements in emulsifier scintillator solution 299 TM from Packard, using a Packard-PRIAS-Tricarb scintillation counter.

Vesicles showed the same ${ }^{3} \mathrm{H} /{ }^{14} \mathrm{C}$ ratio as the starting lipid mixture. Analysis by thin-layer chromatography showed that the lysophosphatidylcholine content was smaller than or equal to 2 mole per cent of the total PC present in the vesicles. Lipid peroxidation of the unsaturated $\mathrm{PC}$ species was 3 to $4 \%$ as determined in a $1-\mathrm{cm}$ quartz cuvette in a Unicam SP 1700 double-beam UV spectrophotometer by measuring the A233/ A215 ratio according to Klein [19].

Preparation of the transfer protein. The phosphatidylcholine specific transfer protein was purified from bovine liver according to Kamp and Wirtz [20], using the modification of Wirtz et al. [21], and had a specific activity of $5 \mu \mathrm{mol} \mathrm{PC}$ exchanged per mg per min. It was homogeneous on SDS-disc gel electrophoresis. The protein was stored at a concentration of $75 \mu \mathrm{g} / \mathrm{ml}$, determined according to Lowry et al. [22], in 50\% glycerol at $-20^{\circ} \mathrm{C}$. Before use, glycerol was removed by dialyzing the protein solution overnight against a 1000 -fold volume of buffer at $4^{\circ} \mathrm{C}$. After dialysis, the protein solution was concentrated against flake polyethylene glycol (Calbiochem, San Diego, U.S.A.).

Erythrocyte preparation. Fresh human erythrocytes were obtained from healthy volunteers by venapuncture. Acid-citrate-dextrose was used as anticoagulant [23]. Cells were packed for $10 \mathrm{~min}$ at $2500 \times \mathrm{g}$ and washed three times with a 4-fold excess of buffer. The buffy coat was carefully removed after each washing.

Incubation conditions. Incubations were carried out at $37^{\circ} \mathrm{C}$ in a thermostated room on a clinical blood rotator at $4 \mathrm{rpm}$. Cells were incubated as a $30-40 \%$ suspension in buffer containing 2 to $3 \mu \mathrm{M}$ transfer protein and $\mathrm{PC}$ donor vesicles. The ratio of vesicle to erythrocyte PC varied between 1 and 4. Aliquots were taken at timed intervals and added to $5 \mathrm{ml}$ buffer of $37^{\circ} \mathrm{C}$ in order to reduce hemolysis by thermal shock. Cells were isolated by centrifugation for $5 \mathrm{~min}$ at $2500 \times \mathrm{g}$ and the resulting supernatant was used to determine hemolysis by measuring the absorbance at $408 \mathrm{~nm}$. Intact cells were examined with respect to the extent of PC replacement, potassium leakage and osmotic fragility.

Phosphatidylcholine replacement. The extent of PC replacement was determined as follows: The cells were washed twice in a 30-fold volume of buffer, which was kept at a temperature of $25-35^{\circ} \mathrm{C}$ in order to minimize contamination of the resulting erythrocyte pellet with vesicles. A third washing had no further effect on the degree of this contamination. Lipids were extracted from the erythrocytes according to the procedure of Rose and Oklander [24] and separated by two-dimensional thin-layer chromatography according to the method of Broekhuyse [25]. The specific radioactivity of the erythrocyte PC was determined using established procedures for determination of phosphorus [29], and radioactivity (see above). This specific activity can be directly correlated with the extent of PC replacement when the specific activity of the donor PC at the start of the incubation is known and when the radioactively labeled PC is representative for the bulk of donor PC. This procedure is based on the theoretical approach of Shipley and Clark [26] and has been applied previously [27].

The extent of contamination of erythrocytes with vesicles is determined by measuring in the lipid extract of the incubated cells the amount of $\left[{ }^{3} \mathrm{H}\right]$ glycerol trioleate, which had been added to the donor system as a non-exchangeable marker. The extent of PC replacement can also be determined by gas-chromatographic analysis of the fatty acyl chains present in the PC of the erythrocyte before and after the replacement procedure. The fatty acyl chains of the isolated erythrocyte PC were converted into their methyl esters according to the method of Morrison and Smith [28]. Gas-liquid chromatographic analysis was performed on a Packard B 24 gaschromatograph, equipped with a Chrompack PB 419 column.

Phosphorus was determined by the method of Rouser et al. [29] and cholesterol as described by Veerkamp and Broekhuyse [30]. 
Potassium leakage determination. In order to measure potassium leakage and content of the cells, they were washed twice at $37^{\circ} \mathrm{C}$ in buffer containing $137.5 \mathrm{mM}$ choline chloride, $17 \mathrm{mM}$ sucrose, $10 \mathrm{mM}$ Tris, $\mathrm{pH} \mathrm{7.4.} 25 \mu 1$ of cells were suspended in $5 \mathrm{ml}$ of this choline chloride buffer at $37^{\circ} \mathrm{C}$ and potassium release was recorded continuously using a potassium selective electrode connected to a Radiometer PHM $26 \mathrm{pH}$ meter. Total potassium content of the cells was determined after lysis by adding $100 \mu 1$ of a $10 \%$ Triton X-100 solution.

Potassium leakage under hypotonic stress was determined by suspending $10 \mu \mathrm{l}$ of cells in $1 \mathrm{ml}$ of buffer, containing $10 \mathrm{mM}$ Tris, $\mathrm{pH} 7.4$ and varying amounts of sodium chloride. After gently mixing for $30 \mathrm{~min}$ at $25^{\circ} \mathrm{C}$, the suspension was centrifuged and sodium and potassium concentrations were measured in the supernatant using a Varian Techtron Atomic Absorption spectrophotometer. Hemolysis was determined from the absorption at $408 \mathrm{~nm}$.

Osmotic fragility. Cells, sampled from the incubation mixture, were washed twice with buffer at $25-35^{\circ} \mathrm{C}$. The osmotic fragility was determined by measuring the change in turbidity of an erythrocyte suspension at $690 \mathrm{~nm}$ as function of salt concentration [31-33]. $10 \mu 1$ of packed cells were suspended in $10 \mathrm{ml}$ of isotonic buffer in a thermostated cuvette. A solution of $10 \mathrm{mM}$ Tris-

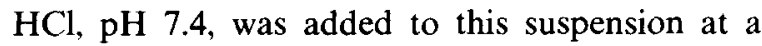
rate of $1 \mathrm{ml} / \mathrm{min}$, using a peristaltic pump of high accuracy. The change in turbidity was recorded continuously. The observed changes were corrected for the effect of dilution of the suspension by repeating the experiment, using isotonic buffer instead of the $10 \mathrm{mM}$ Tris- $\mathrm{HCl}$ solution. The osmotic fragility curves thus obtained appeared to have a high degree of reproducibility.

\section{Results}

When human erythrocytes were incubated at $37^{\circ} \mathrm{C}$ in the presence of phosphatidylcholine specific transfer protein from bovine liver and donor vesicles consisting of equimolar amounts of cholesterol and PC, the PC from the erythrocyte was replaced by the $\mathrm{PC}$ present in the vesicles. This replacement starts progressively but levels off at a plateau after a few hours. Three different types of PC were used in these experiments: 1saturated 2-unsaturated species, found in relatively large amounts in the (human) erythrocyte membrane, and the less common di-saturated and diunsaturated species. The 1-saturated 2-unsaturated species could be exchanged quite rapidly. Fig. 1 shows exchange profiles obtained with 1 palmitoyl-2-oleoyl-, 1-palmitoyl-2-linoleoyl- and 1-palmitoyl-2-arachidonoyl PC. The rate and extent of PC replacement depend on the type of PC species and the amount of donor PC present. In general, a 3-fold excess of donor $\mathrm{PC}$, relative to the amount of PC present in the erythrocytes, appeared to be sufficient to replace about $60 \%$ of the native erythrocyte $\mathrm{PC}$ by each of these 1saturated 2-unsaturated species. This is consistent with the assumption that all of the PC in the outer membrane leaflet is fully available for such a replacement process [34].

Rapid replacement was also observed for the di-unsaturated species dioleoyl and dilinoleoylphosphatidylcholine (Fig. 1B). In the case of dioleoyl PC, a plateau was reached at a replacement level of about $60 \%$ of the total native erythrocyte PC. Replacement of erythrocyte PC by dilinoleoylphosphatidylcholine was followed only until a replacement of about $50 \%$ had been reached. However, this figure should be treated with care due to the extensive hemolysis which starts to take place at replacement levels of about $40 \%$ and higher.

The replacement of native erythrocyte phosphatidylcholine by dipalmitoyl- and distearoyl PC proceeds much more difficult. Under similar conditions as applied to the 1-saturated 2-unsaturated species, only $20-25 \%$ of the erythrocyte PC could be replaced (Fig. 1C). A more extensive replacement of PC was obtained only by repeatedly replacing the donor vesicles by fresh material, as indicated in the figure. This way, up to $65 \%$ of the total erythrocyte PC could be replaced by dipalmitoylphosphatidylcholine.

The phosphatidylcholine donor vesicles used in the experiments described above, contained in addition to the bulk PC also trace amounts of radioactive PC, which were added to facilitate the determinations of the rate and extent of replacement. The data presented in Fig. 1 are based on amounts 


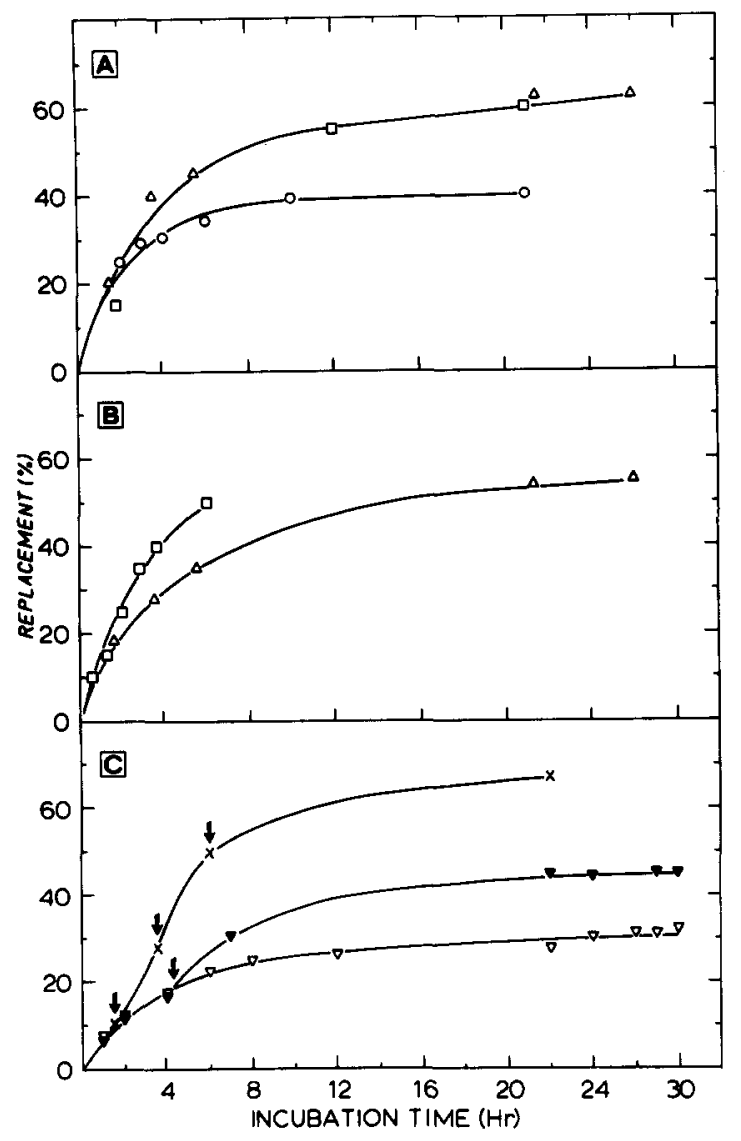

Fig. 1. Replacement of native erythrocyte PC by various PC species. Human erythrocytes were incubated with donor PC vesicles and $\mathrm{PC}$ specific exchange protein as described in Materials and Methods. At timed intervals, erythrocytes were collected and analyzed for their PC content. The extent of PC replacement was determined by using trace amounts of radioactive $P C$ in the donor vesicles and analyzing the resulting specific radioactivity of the erythrocyte $\mathrm{PC}$; data are expressed as $\%$ of total PC present. (A) The replacement of erythrocyte PC by 1-palmitoyl-2-oleoylphosphatidylcholine $(\Delta)$; 1 palmitoyl-2-linoleoylphosphatidylcholine $(O)$ and 1-palmitoyl2-arachidonoylphosphatidylcholine (D). The molar ratio of donor vesicle PC to erythrocyte PC was 3 in case of 1palmitoyl-2-oleoyl PC and 2.4 in the other incubations. (B) Replacement profiles of erythrocyte PC by 1,2-dioleoylphosphatidylcholine $(\Delta)$ and 1,2-di-linoleoylphosphatidylcholine ( $\square$ ) both present in a two-fold excess of the erythrocyte PC. (C) Replacement profiles obtained with 1,2-dipalmitoylphosphatidylcholine. A 2-fold excess of donor PC over erythrocyte $P C$ resulted in this case in limited replacement only $(\nabla)$. When after $4 \mathrm{~h}$ of incubation the cells were isolated, washed as described and reincubated at $37^{\circ} \mathrm{C}$ with a fresh amount of donor PC and exchange protein, more erythrocyte PC could be replaced ( $\nabla)$. Optimal replacement was obtained by repeated refreshing of the donor PC $(X)$ at various time points indicated by the arrows. of radioactive label transferred from donor vesicle to erythrocyte. However, Fig. 1 also shows that the rate and extent by which the replacement takes place are dependent on the phosphatidylcholine species involved and it cannot, therefore, be excluded that the use of a radioactive tracer molecule representing the phosphatidylcholine bulk, results in an over- or underestimation of the actual replacement, when the fatty acyl chain composition of tracer and bulk PC differ.

In order to investigate this possibility, we have analyzed the fatty acyl composition of erythrocyte phosphatidylcholine before and after the replacement reaction and compared these gaschromatographic data with the data based on label incorporation. The results, summarized in Table I, show that radioactive tracer molecules are representative for the phosphatidylcholine bulk only, when their fatty acyl composition is similar to that of the bulk. It is obvious that $\left[{ }^{14} \mathrm{C}\right]$ dipalmitoyl PC can be used as a tracer to monitor routinely the replacement of both dipalmitoyl- and distearoyl PC. On the other hand, an underestimation of the extent of replacement is evident from the experiments in which 1-palmitoyl-2-[ $\left[{ }^{14} \mathrm{C}\right]$ oleoylphosphatidylcholine is used as tracer in vesicles containing 1. palmitoyl-2-arachidonoylphosphatidylcholine as major component. Based on these observations, in all subsequent experiments the extent of PC exchange was measured by using both the radioactive tracer procedure as well as gas-chromatographic quantitation of the relative amounts of the paraffinic side chains of the erythrocyte PC.

To ascertain that the experimental approach resulted only in a one-to-one replacement of erythrocyte phosphatidylcholine and that the observed modifications described below were only due to this replacement, a variety of control experiments was carried out. The extent of contamination of erythrocytes with vesicles after washing could be measured, by including the nonexchangeable marker $\left[{ }^{3} \mathrm{H}\right]$ glycerol trioleate into vesicles. The contamination of erythrocyte phosphatidylcholine with vesicle phosphatidylcholine was normally less than $4 \%$ of the total erythrocyte phosphatidylcholine. Analysis of the erythrocyte phospholipid composition showed that in case of a low degree of contamination, the phosphatidylethanolamine/phosphatidylcholine ratio of the 
TABLE I

FATTY ACID COMPOSITION OF PC FROM HUMAN ERYTHROCYTES AFTER REPLACEMENT WITH VARIOUS PC SPECIES

The PC in intact human erythrocytes was replaced by PC of various fatty acid compositions as described in the text. The donor PC vesicles contained in addition to the bulk PC, radioactive PC in trace amounts. The fatty acid composition of bulk and tracer PC are given in the table; radioactivity is indicated by $\left({ }^{*}\right)$. After the replacement incubation, PC was isolated following the procedures described. Both specific radioactivity and fatty acid composition of the isolated PC were determined and used to calculate the extent of replacement. The calculations on the basis of fatty acid composition were carried out assuming that the replacement involved random removal of the different PC species from the outer layer of the erythrocyte membrane. Data on fatty acid composition are expressed in $\%$ of total fatty acid and replacement data are expressed as $\%$ of total PC present in the erythrocytes.

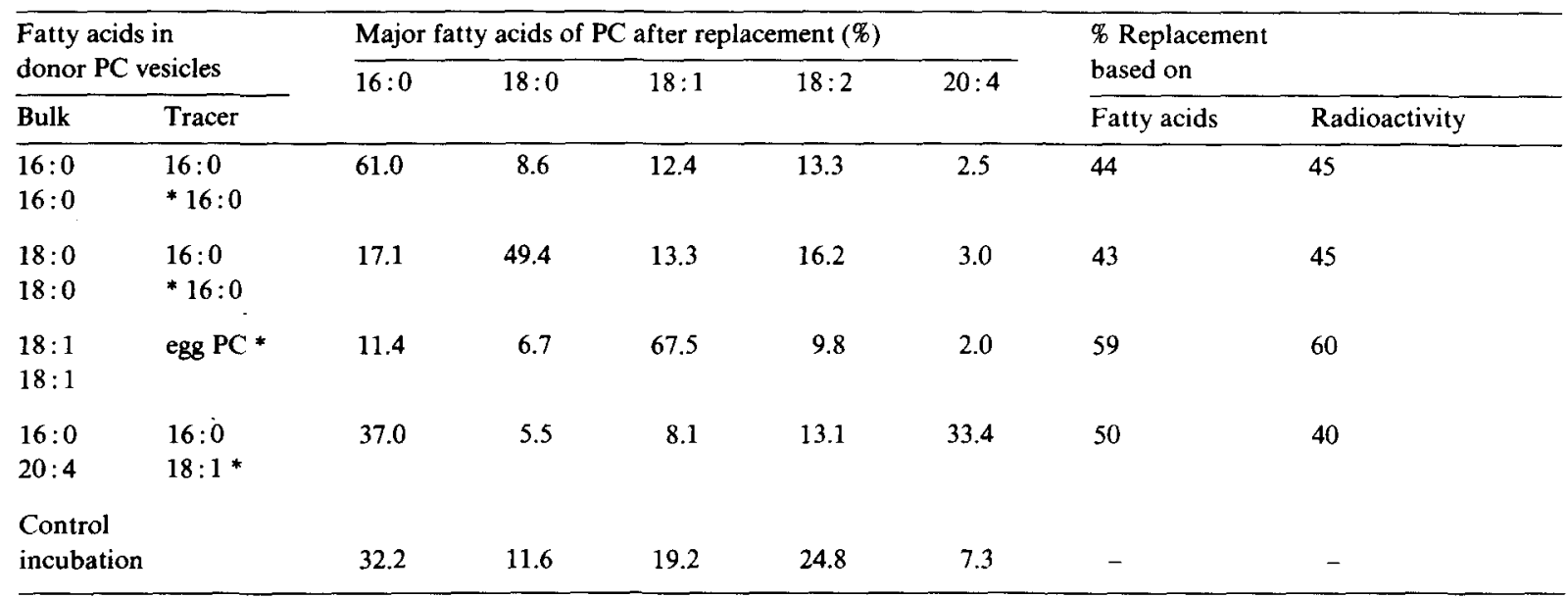

modified erythrocytes did not significantly differ from the non-modified ones (Table II). In addition, the phospholipid/cholesterol ratio and lysophosphatidylcholine content of the modified erythrocytes did not show any significant differences either. Finally, peroxidation of lipids is not thought to be responsible for the observed effects, because control experiments during and after the exchange incubation showed that the extent of peroxidation was less than $3 \%$ and was similar in case of replacement with egg PC, 1palmitoyl-2-linoleoyl- and 1-palmitoyl-2-arachidonoyl PC.

TABLE II

\section{LIPID COMPOSITION OF HUMAN ERYTHROCYTES AFTER REPLACEMENT OF PC}

Various PC species have been used to replace native PC. The extent of replacement is given as $\%$ of total PC present in the membrane. The lipid composition is expressed as molar ratios of phosphatidylcholine: phosphatidylethanolamine (PC/PE) and total phospholipid : cholesterol (PPL/Chol). The content of lysophosphatidylcholine (lyso PC) is expressed as \% of total phospholipid present.

\begin{tabular}{lllll}
\hline PC replacement & & \multicolumn{2}{l}{ Lipid composition } & \\
\cline { 4 - 5 } Species & Extent (\%) & PC/PE & PPL/Chol & Lyso PC (\%) \\
\hline- & - & 1.04 & $0.8 \pm 0.1$ & $2 \pm 1$ \\
$16: 0 / 16: 0$ & 30 & 1.00 & 1.0 & 2 \\
$18: 0 / 18: 0$ & 30 & 1.04 & 0.9 & 0.5 \\
$16: 0 / 18: 1$ & 60 & 1.04 & 0.8 & 2 \\
$16: 0 / 18: 2$ & 60 & 1.03 & 0.85 & 3 \\
$16: 0 / 20: 4$ & 60 & 1.11 & 1.0 & 2 \\
$18: 1 / 18: 1$ & 60 & 1.08 & 0.9 & 2 \\
$18: 2 / 18: 2$ & 30 & 1.07 & 0.95 & 2 \\
\hline
\end{tabular}


Effect on osmotic fragility and membrane permeability for potassium

The structure of the fatty acyl residues which form the apolar core of the membrane, determine to a large extent the interactions between the various membrane constituents and in this way, the permeability and mechanical strength of the membranes. An estimation of these parameters can be obtained by measuring potassium leakage and osmotic fragility, respectively. We therefore studied these two phenomena in their relation to the phosphatidylcholine replacement of the native erythrocyte phosphatidylcholine.

In our experimental set-up control cells start to lyse in a buffer in which the osmolarity is about $60 \%$ of the isotonic value, $50 \%$ of the cells is lysed at an osmolarity of about $45 \%$ and lysis is complete at an osmolarity of $40 \%$. In our continuous dilution method we find, like other authors [31,32], a shift of the osmotic fragility curve to the hypotonic side, when compared to the classical discontinuous method. Control experiments showed that incubation up to $21 \mathrm{~h}$ under the conditions used for the PC replacement gives only a slight shift in osmotic fragility of the cells (Fig. 2). The replacement of native erythrocyte phosphatidylcholine by the disaturated species dipalmitoylphosphatidylcholine and distearoylphosphatidylcholine, results in a shift in the osmotic fragility to the isotonic side, demonstrating that the cells become more fragile towards hypotonic stress (Fig. 2). This increase in fragility starts at about $20 \%$ of replacement, and parallels the extent of replacement thereafter. However, above $40 \%$ of replacement, cells start to lyse already in the isotonic incubation buffer (Fig. 3). Passive potassium leakage in isotonic buffer is not significantly different from control cells up to a replacement of $30 \%$ of the native erythrocyte phosphatidylcholine (Table III).

Replacement of native erythrocyte phosphatidylcholine with the saturated-unsaturated species 1-palmitoyl-2-oleoylphosphatidylcholine and 1palmitoyl-2-linoleoylphosphatidylcholine had no effect on potassium leakage, nor on osmotic fragility of the cells (Fig. 2). Also replacement by the di-unsaturated dioleoylphosphatidylcholine had no effect on osmotic fragility up to about $40 \%$. At higher replacement levels however, a slight shift was observed (Fig. 2), but an enhanced leakage of

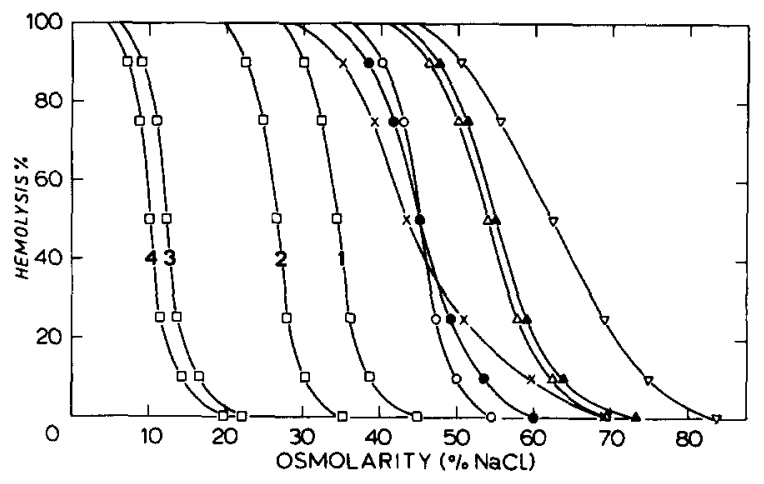

Fig. 2. Effect of replacement of native erythrocyte $\mathrm{PC}$ on osmotic fragility. PC from human erythrocytes has been replaced by various $\mathrm{PC}$ species following the incubation conditions as described in the legend to Fig. 1, and Materials and Methods. After incubation, cells were isolated, washed as described and the osmotic fragility was determined, using the continuous dilution method described in the text. The extent of hemolysis (expressed as $\%$ of total cells lysed) is plotted against the osmolarity (expressed as $\%$ of the isotonic value). The following control and replacement experiments are represented: Control cells $(O)$ and control cells after $21 \mathrm{~h}$ of incubation ( $\bullet$ ). Identical results as given by $(O)$ were obtained with cells in which $60 \%$ and $40 \%$ of the native PC species were replaced by 1-palmitoyl-2-oleoylphosphatidylcholine and 1palmitoyl-2-linoleoylphosphatidylcholine, respectively. Cells in which $20 \%(\square, 1) ; 40 \%(\square, 2) ; 55 \%(\square, 3)$ and $60 \%(\square, 4)$ of native $P C$ was replaced by 1-palmitoyl-2-arachidonoylphosphatidylcholine. Cells in which $40 \%$ of the native PC was replaced by 1,2-dipalmitoylphosphatidylcholine $(\Delta)$ and 1,2-distearoylphosphatidylcholine ( $\triangle$ ). Cells in which $65 \%$ of the native PC was replaced by 1,2-dioleoylphosphatidylcholine $(X)$. Cells in which $25 \%$ of the native PC was replaced by 1,2-dilinoleoylphosphatidylcholine $(\nabla)$.

potassium from the cells could still not be detected. In contrast to this, replacement with dilinoleoylphosphatidylcholine leads to a drastic modification. The osmotic fragility is apparently increased and these cells are also more leaky to potassium in isotonic buffer than normal cells. Furthermore, hemolysis in isotonic buffer was observed at a replacement exceeding $25 \%$ (Fig. 3). The opposite effect was observed with 1-palmitoyl2 -arachidonoylphosphatidylcholine. Concomitant with the extent of replacement of native erythrocyte phosphatidylcholine by this species, there is an apparent decrease in osmotic fragility (Fig. 2). Potassium leakage under isotonic conditions is slightly enhanced compared to native cells, but the modified cells loose their potassium content much 


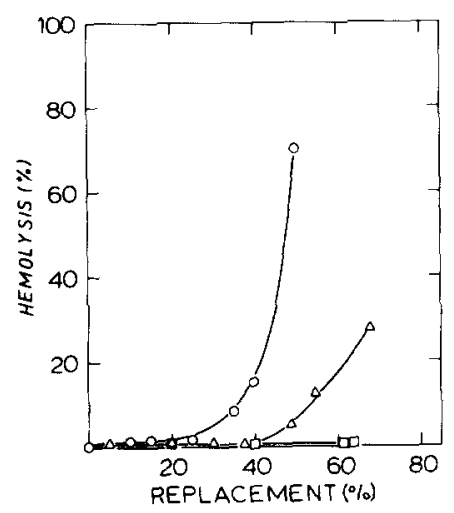

Fig. 3. Hemolysis of erythrocytes following PC replacement. Cells were incubated as described in the legend to Fig. 1 with 1,2-dipalmitoyl PC ( $\Delta), 1,2$-dilinoleoyl PC $(O)$ or 1-palmitoyl2-oleoyl PC ( $\square$ ) vesicles. The hemolysis was measured after increasing replacement of native $P C$ by these species and determined from the absorbance at $408 \mathrm{~nm}$ of the reaction supernatant after centrifugation. Data are expressed as $\%$ of total hemoglobin present. Data with respect to the replacement are expressed as $\%$ of total PC present in the erythrocyte.

faster under hypotonic conditions (Table III; Fig. 4).

Potassium leakage under hypotonic conditions has been compared with hemoglobin release under similar conditions. Cells were suspended in media of different tonicity and the release of both compounds was recorded. The data in Fig. 4 show that, as is known, $\mathrm{K}^{+}$release preceeds hemoglobin

\section{TABLE III}

\section{LEAKAGE OF $\mathrm{K}^{+}$FROM MODIFIED HUMAN ERYTHROCYTES}

The extent of PC replacement is presented as $\%$ of total PC present in the membrane. The $\mathrm{K}^{+}$leakage data are expressed as $\mathscr{6}$ of total $\mathrm{K}^{+}$present in the erythrocyte, released per $15 \mathrm{~min}$ of incubation under the conditions described in Materials and Methods.

\begin{tabular}{|c|c|c|}
\hline \multicolumn{2}{|l|}{ Replacement } & \multirow{2}{*}{$\begin{array}{l}\text { Rate of } \mathrm{K}^{+} \\
\text {leakage }\end{array}$} \\
\hline Species & Extent $(\mathscr{6})$ & \\
\hline- & - & $0.2 \pm 0.1$ \\
\hline $16: 0 / 16: 0$ & 30 & 0.4 \\
\hline $18: 0 / 18: 0$ & 30 & 0.4 \\
\hline $16: 0 / 18: 1$ & 60 & 0.3 \\
\hline $16: 0 / 18: 2$ & 60 & 0.3 \\
\hline $16: 0 / 20: 4$ & 60 & 2.0 \\
\hline $18: 1 / 18: 1$ & 60 & 0.3 \\
\hline $18: 2 / 18: 2$ & 30 & 2.5 \\
\hline
\end{tabular}

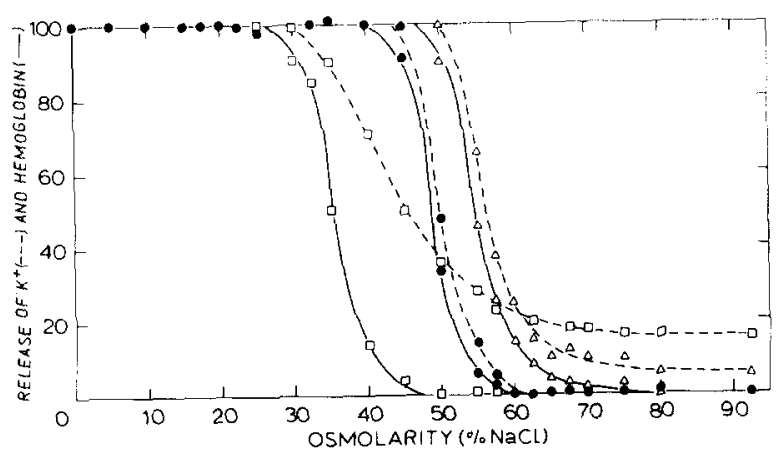

Fig. 4. Effect of replacement of native erythrocyte $\mathrm{PC}$ on $\mathrm{K}^{+}$ and hemoglobin permeability. The PC of intact human erythrocytes has been replaced by other PC species as described in the legend to Fig. 1 and Materials and Methods. After incubation, the cells were washed as described and analyzed for the extent of PC replacement. Cells were analyzed furthermore for their $\mathrm{K}^{+}$content and the permeability of the membrane for $\mathrm{K}^{+}$and hemoglobin. The discontinuous dilution method described in the Materials and Methods section was applied to measure hemoglobin permeability. The figure shows the release of $\mathrm{K}^{+}$(dotted line) and hemoglobin (straight line), expressed as $\%$ of total $\mathrm{K}^{+}$and hemoglobin, present, respectively, as function of osmolarity (expressed as $\%$ of the isotonic value of $300 \operatorname{mos} \mathrm{M})$. Control cells $(\bullet)$, cells in which $30 \%$ of the native PC was replaced by dipalmitoyl PC $(\Delta)$ and cells in which $20 \%$ of the PC was replaced by 1-palmitoyl-2arachidonoyl PC ( $\square$ ), are shown. The curves obtained for control cells are identical with the curves obtained with cells in which $40 \%$ of the native PC was replaced by 1-palmitoyl-2oleoyl PC (ब).

release upon lowering the tonicity. This is obvious for control cells and also for cells in which PC has been replaced by 1-palmitoyl-2-oleoyl PC. However, after replacement with 1,2-dipalmitoyl PC, the shift in the osmotic fragility curve (cf. also Fig. 2) parallels that of the curve recorded for the $K^{+}$ leakage. Completely different results were obtained after replacement with 1-palmitoyl-2arachidonoyl PC. The primary defect induced here is that the membrane looses its barrier properties, especially for ions. $\mathrm{K}^{+}$leaks out of the cell already at isotonic conditions and it is reasonable to assume that $\mathrm{Na}^{+}$can move in as freely. Due to this loss in barrier properties, the osmotic resistance curve is shifted to the hypotonic side (Fig. 4).

\section{Discussion}

The phosphatidylcholine specific exchange protein from bovine liver has been applied to modify 
the fatty acyl species composition of phosphatidylcholine in intact human erythrocytes.

The species-dependent rate difference in the exchange process observed in this study has been noted before and has been investigated in detail using various model systems [35-37]. The reason for this species difference has been sought in the specificity of the protein itself as well as in the physico-chemical properties of the donor and acceptor membrane systems $[37,38]$. In the studies presented here, two additional complicating factors have to be taken into account. First of all, donor vesicles have been used which contain equimolar amounts of PC and cholesterol and the effects of such a high concentration of cholesterol on the exchange process was not studied before. Secondly, the donor PC vesicles are modified during the exchange process with a heterogenous PC mixture originating from the erythrocytes and it is again not known how this affects exchange rates. The effect which such a modification may have is most obvious from the experiments with the dipalmitoyl PC vesicles as donor systems. After a certain amount of PC has been replaced, the composition of the vesicles is changed in such a way that exchange of dipalmitoyl PC itself is slowed down considerably. However, exchange continues as is indicated by experiments in which different, unsaturated PC species are present in small amounts as probe molecules in the disaturated bulk (unpublished results). It is most likely, therefore, that during the ongoing exchange between the modified donor vesicles and the erythrocytes, a preferential transfer of unsaturated PC molecules occurs.

This preference of the exchange protein seems to be underlined by the observation that the erythrocyte is not the only limiting factor because it can, in principle, accept more disaturated PC molecules after replacing the modified donor vesicles by fresh material (Fig. 1C).

The effect of PC replacement on the properties of the membrane of the erythrocytes (summarized in Table IV) can be quite dramatic. Before discussing these effects in detail, however, it is worthwhile to emphasize that replacement with a number of PC species has no effect at all. Palmitoyloleoyl PC and palmitoyllinoleoyl PC, as well as egg PC and PC from rat liver microsomes (data not shown), are perfectly capable to replace the native erythrocyte PC without any noticeable effect on membrane permeability or stability. This type of mixed acid PC species in which one saturated fatty acid and one unsaturated fatty acid containing one or two double bonds, are present, is the most common PC in the human erythrocyte membrane and comprises up to $60 \%$ of the total species composition [3]. It is obvious that this PC offers the most favourable configuration for a stable membrane and that both increasing or decreasing the degree of unsaturation of the PC results in abnormalities.

An increase in disaturated PC species results in an increased fragility of the human erythrocyte membrane which finally, after $40 \%$ replacement, results in hemolysis. A similar observation has been made before with rat erythrocytes [16], although in this case hemolysis started already after $20 \%$ replacement. Rat erythrocyte PC contains, however, a high content (35\%) of disaturated species, whereas in human erythrocytes only $13 \%$ of the total PC is present in the disaturated form. The results for both red cell species is therefore similar: when about $40-50 \%$ of the total PC content is in a disaturated form, it cannot stabilize the membrane sufficiently any more and hemolysis starts to take place. The human erythrocyte contains PC which has an unsaturation index close to 1. The index, which is calculated as $1 / 100 \times$ (the amount of double bonds present per 50 PC molecules) can be decreased therefore to 0.5 without noticeable effects on membrane structure and function. However, below this value, membrane stability is decreasing as is evident from the replacement with disaturated PC. Upon an increase in the unsaturation index, the permeability is increased and therefore an index which lies in between 0.5 and 1.0 seems to be optimal for PC under the conditions described here. Phosphatidylcholine accounts for about $30 \%$ of the total phospholipid fraction, which is about $15 \%$ of the total lipids of the erythrocyte membrane. Dipalmitoyl PC accounts for only $2 \%$ of the total lipid. It is remarkable to notice how a very small increase in the relative amount of this particular lipid species from $2 \%$ to $4 \%$ of the total lipid complement, has large effects on the membrane stability and finally, at a relative concentration of $6 \%$, results in hemolysis. 
TABLE IV

\section{EFFECTS OF CHANGES IN THE SPECIES COMPOSITION OF PC IN HUMAN ERYTHROCYTES}

The effects of the replacement of native PC from intact human erythrocytes by a variety of PC species differing in fatty acid composition is summarized. PC species have been used which are already present in the erythrocytes (indicated by ${ }^{*}$ ), as well as species which normally do not occur (18:0/18:0 and 18:2/18:2 PC) and mixtures of PC species like egg PC and PC from rat liver microsomal membranes. The increase of a species content in the erythrocyte membrane is expressed as $\mathscr{t}$ of total PC.

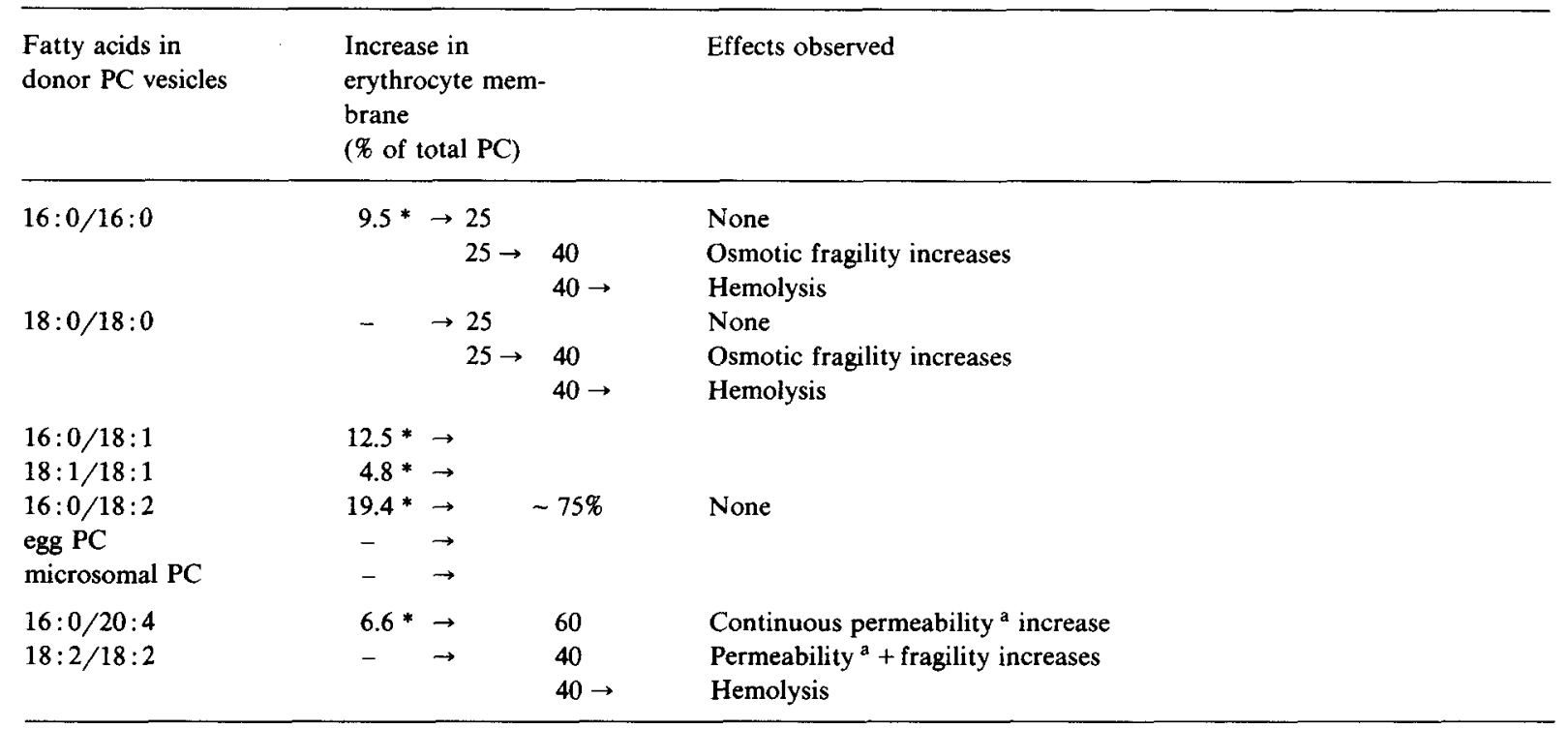

a Permeability of the membrane determined as $\mathrm{K}^{+}$leakage from the cells under isotonic conditions.

* Data taken from Ref. 3.

The molecular mechanism responsible for the observed increases in osmotic fragility and permeability are not clear. However, studies on model membrane systems, in particular those describing effects of cholesterol on membrane structure and properties, may give a (partial) explanation of some of the phenomena observed in these studies with erythrocytes. Monolayer studies showed that there is no condensing effect of cholesterol on the packing of dipalmitoyl PC and dilinoleoyl PC $[39,40]$. This implies that replacement of $P C$ species which can interact optimally with cholesterol in the erythrocyte membrane by molecules which interact less efficiently, weakens the coherence of membrane constituents, which is expressed by an increased osmotic fragility. On the other hand, it is known that a high degree of unsaturation enhances permeability and cholesterol does not diminish this in case of dilinoleoyl PC and palmitoyl-arachidonoyl PC [40].

Although these considerations are relevant, it has to be emphasized that other parameters such as the interaction of PC with membrane constituents like phosphatidylethanolamine, sphingomyelin as well as intrinsic proteins, may also contribute to the overall stability and permeability of the system. Finally, it has to be realized that the modifications in lipid/lipid and lipid/protein interactions which are achieved by the replacement reaction, are induced in the outer layer of the membrane only. Ample evidence has been produced, demonstrating a slow transbilayer movement of phospholipid in the erythrocyte membrane $[27,41]$, and it is obvious therefore, that the equilibrium in structure and properties between outer and inner layer of the membrane can be disturbed under our conditions.

In order to understand the specific effects on membrane structure and function caused by changes in fatty acid composition, studies have been carried out previously on erythrocytes in which changes were induced by supplementation 
of different diets to various mammals, including man. The results of such studies show that large differences in dietary composition resulted in rather limited alterations in the phospholipid composition of the erythrocyte (see Refs. 10 and 13 for detailed description).

A comparison between the in vivo changes and the alterations brought about by the exchange experiments described in this study, suggests clearly that the tolerance of the erythrocyte to undergo changes in lipid structure is very limited and that apparently effective systems must operate in vivo, to keep such diet induced changes within acceptable limits, in order to maintain the optimal functioning of the red cell membrane. The well documented low turnover rate of phospholipids in the erythrocyte membrane will certainly attribute to this phenomenon $[14,15]$.

\section{Acknowledgements}

The present investigations were carried out under the auspices of The Netherlands Foundation for Chemical Research (SON) and with financial aid from the Netherlands Organization for the Advancement of Pure Research (ZWO). We wish to thank Mrs. M. van Linde for the preparation of highly purified exchange protein.

\section{References}

1 Van Deenen, L.L.M. and De Gier, J. (1974) in The Red Blood Cell, Vol. 1, 2nd Edn. (Surgenor, D.M., ed.), pp. 147-211, Academic Press, New York

2 Dodge, J.T. and Phillips, G.B. (1967) J. Lipid Res. 8, 667-675

3 Van Golde, L.M.G., Tomasi, V. and Van Deenen, L.L.M. (1967) Chem. Phys. Lipids 1, 282-293

4 Marinetti, G.V. and Crain, R.C. (1978) J. Supramol. Struct. 8, 191-213

5 Verkleij, A.J., Zwaal, R.F.A., Roelofsen, B., Comfurius, P., Kastelijn, D. and Van Deenen, L.L.M. (1973) Biochim. Biophys. Acta 323, 178-193

6 Renooij, W., Van Golde, L.M.G., Zwaal, R.F.A., Roelofsen, B. and Van Deenen, L.L.M. (1974) Biochim. Biophys. Acta 363, 287-292

7 Renooij, W., Van Golde, L.M.G., Zwaal, R.F.A. and Van Deenen, L.L.M. (1976) Eur. J. Biochem. 61, 53-58

8 Marinetti, G.V. and Cattieu, K. (1982) J. Biol. Chem. 257, 245-248

9 Phillips, S.B., Dodge, J.T. and Howe, C. (1969) Lipids 4, 544-549

10 De Gier, J. (1968) Bibl. Haematol. 29, 158-165

11 Shand, J.H. and Noble, R.C. (1981) Biol. Neonate 40, 150-159
12 Vajreswari, A., Srinivasa Rao, P., Kaplay, S.S. and Tulpule, P.G. (1983) Biochem. Med. 29, 74-84

13 Ehrström, M., Harms-Ringdol, M. and Alling, $\dot{C}$. (1981) Biochim. Biophys. Acta 644, 175-182

14 Renooij, W. (1977) Ph.D. Thesis, State University of Utrecht, The Netherlands

15 Shohet, S.B. (1970) J. Clin. Invest. 49, 1668-1678

16 Lange, L.G., Van Meer, G., Op den Kamp, J.A.F. and Van Deenen, L.L.M. (1980) Eur. J. Biochem. 110, 115-121

17 Van Deenen, L.L.M. and De Haas, G.H. (1964) Adv. Lipid Res. 29, 168-229

18 Stoffel, W. (1928) Methods Enzymol. 35, 533-541

19 Klein, R.A. (1970) Biochim. Biophys. Acta 210, 485-489

20 Kamp, H.H. and Wirtz, K.W.A. (1974) Methods Enzymol. $32,140-146$

21 Wirtz, K.W.A., Vriend, G. and Westerman, J. (1979) Eur. J. Biochem. 94, 215-221

22 Lowry, O.H., Rosebrough, N.J., Farr, A.L. and Randall, R.J. (1951) J. Biol. Chem. 193, 265-275

23 Anderson, H.M. and Turner, J.C. (1960) J. Clin. Invest. 39, 1-7

24 Rose, H.A. and Oklander, M. (1965) J. Lipid Res. 6, 428-431

25 Broekhuyse, R.M. (1969) Clin. Chim. Acta 23, 457-463

26 Shipley, R.A. and Clark, R.E. (1972) Tracer Methods for In Vivo Kinetics-Theory and Applications, p. 132, Academic Press, New York

27 Van Meer, G. and Op den Kamp, J.A.F. (1982) J. Cell Biochem. 19, 193-204

28 Morrison, W.R. and Smith, I.M. (1964) J. Lipid Res. 5, 600-608

29 Rouser, G., Fleischer, S. and Yamamoto, A. (1970) Lipids $5,494-496$

30 Veerkamp, J.H. and Broekhuyse, R.M. (1976) in Biochemical Analysis of Membranes (Maddy, A.H., ed.), pp. 252-258, Wiley, New York

31 Livne, A. and Raz, A. (1971) FEBS Lett. 16, 99-101

32 Seeman, P., Sauks, T., Argent, W. and Kwant, W.O. (1969) Biochim. Biophys. Acta 183, 476-489

33 Maeda, M., Aono, K., Sekiya, M., Suda, T. and Shiga, T. (1977) Anal. Biochem. 83, 149-161

34 Van Meer, G., Poorthuis, B.J.H.M., Wirtz, K.W.A., Op den Kamp, J.A.F. and Van Deenen, L.L.M. (1980) Eur. J. Biochem. 103, 283-288

35 Kamp, H.H., Wirtz, K.W.A., Baer, P.R., Slotboom, A.J., Rosenthal, A.F., Paltauf, F. and Van Deenen, L.L.M. (1977) Biochemistry 16, 1310-1316

36 Schulze, G., Jung, K., Kunze, D. and Egger, E. (1977) FEBS Lett. 74, 220-224

37 Bozzato, R.P. and Tinker, D.O. (1982) Can. J. Biochem. 60, 409-418

38 Kasper, A. and Helmkamp, G.M. (1981) Biochemistry 20, 146-151

39 Demel, R.A., Geurts van Kessel, W.S.M. and Van Deenen, L.L.M. (1972) Biochim. Biophys. Acta 266, 26-40

40 Demel, R.A. and De Kruijff, B. (1976) Biochim. Biophys. Acta 457, 109-132

41 Renooij, W. and Van Golde, L.M.G. (1977) Biochim. Biophys. Acta 470, 465-474

42 Boegheim, J.P.J., Jr., Van Linde, M., Op den Kamp, J.A.F. and Roelofsen, B. (1983) Biochim. Biophys. Acta 735, 438-442 